\title{
Fast Eigen Matching
}

Accelerating Matching and Learning of Eigenspace method

Yusuke Sekikawa, Koichiro Suzuki, Kosuke Hara, Yuichi Yoshida, Ikuro Sato

\{ysekikawa,ksuzuki,khara,yyoshida, isato\}@d-itlab.co.jp

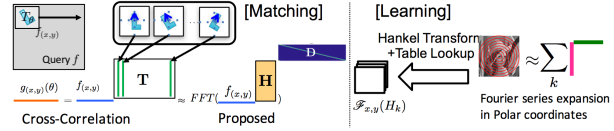

Fig. 1: Overview of the proposed method

We propose Fast Eigen Matching, a method for accelerating the matching and learning processes of the eigenspace method for rotation invariant template matching (RITM).

Correlation-based template matching is one of the basic techniques used in computer vision. Among them, rotation invariant template matching (RITM), which locates a known template in a query irrespective of the template's translation and orientation, has been widely put to use in many industrial applications. A naive implementation of RITM requires intensive computation since one needs to correlate query $f$ with $N$ rotated templates $\boldsymbol{T}$ (Fig.1 left). Eigenspace methods takes advantage of the fact that a set of correlated images $\boldsymbol{T}$ can be approximately represented by a small set of eigenimages. Once eigenimages and it's 2D-Fourier transform are computed in learning process, matching process of RITM can be performed very efficiently using these 2D-Fourier transformed eigenimages [1].

It is also important to speedup the learning process, especially for applications such as global robot localization, where a template changes frame by frame and efficient online learning is required. The existing eigenspace methods are not feasible for problem settings of this kind, because it requires a lot of time for generation of rotated templates, SVD and 2DFFT.

To speed up the matching and the learning process of existing Eigenspace methods, we propose Fast Eigen Matching by exploiting FFT and Hankel Transform. Our contributions are as follows:

Speedup the Matching process By focusing on the circularity of in-plane rotation and con-

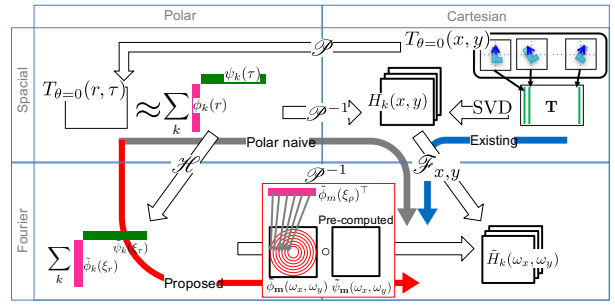

Fig. 2: The learning algorithm.

centration of power spectrums to low frequency, we compute fast-eigenimages $\boldsymbol{H}$ by expanding a templates using Fourier basis, which leads to the use of FFT in a matching process (Fig.1 left).

Speedup the Learning process By utilizing the fact that Fourier expansion in polar coordinates is efficiently transformed to frequency domain using Hankel transform[2], our method computes 2D-Fourier transform of each fasteigenimages $\tilde{\boldsymbol{H}}$ in polar coordinate (Fig.1 right Fig.2). This computation is equivalent to existing learning method, i.e., time-consuming rotated template generation, numerical SVD and 2D-FFTs in Cartesian Coordinates, but substantially boosts the learning process by avoiding these time-consuming computation.

Our experiments revealed that the learning, matching, and total processes respectively becomes 120, 3, and 36 times faster, while keeping comparable matching performance compared to previous method. As a representative example, we show an application to global localization with a Particle Filter.

[1] Gou Koutaki and Keiichi Uchimura. Occlusion Robust Pattern Matching Using Shape Based Eigen Templates. IEEJ Transactions on Electronics, Information and Systems, 133(1):134-141, 2013.

[2] Robert Piessens. The hankel transform. 2000. 